\title{
La adicción como enfermedad: ¿mitigando 0 alimentando el estigma?
}

\section{Addiction as a disease: Mitigating or fuelling stigma?}

\author{
Joan Trujols*,**; Saiko Allende*,**. \\ * Unitat de Conductes Addictives, Servei de Psiquiatria, Hospital de la Santa Creu i Sant Pau, Institut d'Investigació Biomèdica \\ Sant Pau (IIB Sant Pau), Barcelona, España; ** Centro de Investigación Biomédica en Red de Salud Mental (CIBERSAM), \\ Instituto de Salud Carlos III (ISCIII), Madrid, España.
}

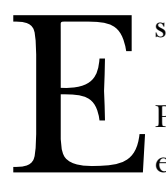

stimado Editor

Recientemente, el editorial publicado por Pascual Mollá y Pascual Pastor (2017) sobre el estigma en la persona adicta se ha hecho eco del plan de sensibilización implementado por Socidrogalcohol, a lo largo de 2017 y en todo el territorio nacional, con el objetivo de reducir la estigmatización de las personas con trastornos adictivos. Quisiéramos felicitar tanto a los autores por su oportuno editorial como a Socidrogalcohol por su pertinente y necesaria iniciativa (más aun si consideramos que las asociaciones científicas no se caracterizan habitualmente por un especial entusiasmo por la acción sociopolítica de carácter público). No obstante, y a propósito tanto de uno de los objetivos de dicho programa de sensibilización ("Aumentar el conocimiento social sobre que la adicción es una enfermedad") como de una de las afirmaciones de dicho editorial ("En cierta forma esta problemática se palió al ser considerados los adictos como enfermos"; Pascual Mollá y Pascual Pastor, 2017, p. 224), nos gustaría recordar que el potencial desestigmatizador del modelo de la adicción como enfermedad no es tan manifiesto como dicho editorial e iniciativa dejan entrever.

Un reducido número de estudios empíricos ha explorado ya esta cuestión. Una encuesta australiana sobre actitudes de la población general ha mostrado que conceptualizar la adicción como una enfermedad cerebral no está asociado a una menor estigmatización ni a un menor apoyo para el tratamiento involuntario u otros abordajes punitivos de la adicción (Meurk, Carter, Partridge, Lucke y Hall, 2014). En esta misma línea, un estudio experimental realizado por Wiens y Walker (2015) ha concluido que el fortalecimiento de la creencia en un modelo de adicción como enfermedad cerebral no sólo no disminuye los sentimientos de estigma y vergüenza en personas con una dependencia leve-moderada del alcohol sino que incluso afecta negativamente varias de sus percepciones de agencia sobre la conducta de beber (p.ej., locus de control, estilo de afrontamiento y niveles de autoeficacia para el beber controlado). Asimismo, un estudio cualitativo sobre las implicaciones de conceptualizar la dependencia de nicotina como una enfermedad cerebral ha mostrado que la mayoría de fumadores entrevistados atribuían connotaciones negativas al témino enfermedad y consideraban que la etiqueta de enfermedad cerebral incrementaba el estigma y prejuicios contra los fumadores (Morphett, Carter, Hall y Gartner, 2017). Algunos participantes referían incluso que asumir un modelo biomédico podría obstaculizar la búsqueda de tratamiento y desanimar eventuales intentos de abandono del consumo de tabaco (Morphett et al., 2017). Finalmente, un estudio experimental en población general recientemente publicado ha mostrado que la atribución de la etiología de la adicción a causas genéticas disminuye significativamente (comparativamente a la atribución a causas no genéticas) los niveles tanto de agencia personal como de autocontrol sobre la conducta adictiva, siendo dichos efectos independientes del tipo de adicción

Recibido: Diciembre 2017; Aceptado: Diciembre 2017.

Enviar correspondencia a:

Joan Trujols. Unitat de Conductes Addictives. Servei de Psiquiatria. Hospital de la Santa Creu i Sant Pau. Sant Antoni Maria Claret 167.08025 Barcelona. E-mail: jtrujols@santpau.cat 
considerada (al alcohol o al juego) en el estudio (Lebowitz y Appelbaum, 2017)

La literatura, bastante más abundante, que vincula estigma y la conceptualización de diagnósticos psiquiátricos en términos biogenéticos o de enfermedad cerebral parece apuntar de forma razonablemente consistente en la misma dirección (Angermeyer, Holzinger, Carta y Schomerus, 2011; Kvaale, Haslam y Gottdiener, 2013). De hecho, desde la perspectiva de la sociología de la salud y la enfermedad, la idea de que el hecho de etiquetar algo como enfermedad mitigará el estigma no deja de ser sorprendente (Fraser et al., 2017).

En cualquier caso, cabe recordar que los orígenes del modelo de la adicción como enfermedad pueden trazarse hasta principios del siglo XVII (Warner, 1994) y que dicho modelo, aunque dominante en las principales asociaciones y publicaciones científicas del ámbito de las adicciones, no es hegemónico entre los trabajadores de dicho ámbito (Trujols, Manresa, Batlle, Duran-Sindreu y Pérez de los Cobos, 2016).

Estas consideraciones no restan importancia a la oportunidad y pertinencia del citado editorial (Pascual Mollá y Pascual Pastor, 2017) ni a la necesidad y relevancia de la iniciativa liderada por Socidrogalcohol, sino que buscan llamar la atención sobre un aspecto concreto que pudiera contribuir a fomentar creencias que perpetuarían más que modificarían actitudes estigmatizantes hacia las personas con problemas de adicción.

\section{Conflicto de intereses}

Los autores declaran que no existe ningún conflicto de intereses relacionado directa o indirectamente con el contenido de este artículo.

\section{Referencias}

Angermeyer, M.C., Holzinger, A., Carta, M.G. y Schomerus, G. (2011). Biogenetic explanations and public acceptance of mental illness: systematic review of population studies. British Journal of Psychiatry, 199, 367-372. doi:10.1192/bjp.bp.110.085563.

Fraser, S., Pienaar, K., Dilkes-Frayne, E., Moore, D., Kokanovic, R., Treloar, C. y Dunlop, A. (2017). Addiction stigma and the biopolitics of liberal modernity: A qualitative analysis. International Journal of Drug Policy, 44, 192201. doi:10.1016/j.drugpo.2017.02.005.

Kvaale, E.P., Haslam, N. y Gottdiener, W.H. (2013). The 'side effects' of medicalization: a meta-analytic review of how biogenetic explanations affect stigma. Clinical Psychology Review, 33, 782-794. doi:10.1016/j. cpr.2013.06.002.

Lebowitz, M.S. y Appelbaum, P.S. (2017). Beneficial and detrimental effects of genetic explanations for addic- tion. International Journal of Social Psychiatry, 63, 717-723. doi:10.1177/0020764017737573.

Meurk, C., Carter, A., Partridge, B., Lucke, J. y Hall, W. (2014). How is acceptance of the brain disease model of addiction related to Australians' attitudes towards addicted individuals and treatments for addiction? $B M C$ Psychiatry, 14, 373. doi:10.1186/s12888-014-0373-x.

Morphett, K., Carter, A., Hall, W. y Gartner, C. (2017). Framing tobacco dependence as a "brain disease": Implications for policy and practice. Nicotine $\mathcal{E}$ Tobacco Research, 19, 774-780. doi:10.1093/ntr/ntx006.

Pascual Mollá, M. y Pascual Pastor, F. (2017). El estigma en la persona adicta. Adicciones, 29, 223-226. doi:10.20882/ adicciones.1038.

Trujols, J., Manresa, M.J., Batlle, F., Duran-Sindreu, S. y Pérez de los Cobos, J. (2016). Deep brain stimulation for addiction treatment: Further considerations on scientific and ethical issues. Brain Stimulation, 9, 788-789. doi:10.1016/j.brs.2016.05.007.

Warner, J. (1994). "Resolv'd to drink no more": addiction as a preindustrial construct. Journal of Studies on Alcohol, 55, 685-691. doi:10.15288/jsa.1994.55.685.

Wiens, T.K. y Walker, L.J. (2015). The chronic disease concept of addiction: helpful or harmful? Addiction Research Ev Theory, 23, 309-321. doi:10.3109/16066359.2014.987 760. 